\title{
Effects of Low and High Doses of Atorvastatin on Arterial Compliance
}

\author{
Yesari KARTER, ${ }^{1}$ MD, Asli ÇURGUnLU, ${ }^{1}$ MD, Nurver ERTÜrK, ${ }^{2}$ MD, \\ Suphi VEHID, ${ }^{3} \mathrm{MD}$, İsmail MiHMANLI, ${ }^{4} \mathrm{MD}$, and Faruk AYAN, ${ }^{5} \mathrm{MD}$
}

\begin{abstract}
SUMMARY
At the beginning of atherosclerosis before evidence of morphological lesions or plaques, vascular distensibility or arterial compliance decreased gradually. This endothelial dysfunction is regarded as an early feature of atherosclerosis. In a randomized, double-blind study design, group I (12 patients; 7 males, 5 females) with serum LDL-C levels higher than $170 \mathrm{mg} / \mathrm{dL}$ and without any other risk factor for atherosclerosis received three months of $20 \mathrm{mg} /$ day atorvastatin treatment while group II ( 8 males, 4 females) with the same characteristics received $80 \mathrm{mg} /$ day. Baseline and posttreatment serum lipid fractions and arterial compliance were measured. Arterial compliance was measured noninvasively in the left common carotid artery with color Doppler ultrasound. Atorvastatin reduced total cholesterol (TC), LDL-C, and triglyceride levels by 32\% $(P<0.001), 40.8 \%(P<$ $0.001)$, and $19 \%(P<0.001)$, respectively, and increased HDL-C by $6.9 \%(P=0.002)$ in the first group. In the second group these reductions were $38.5 \%(P<0.001), 46.2 \%(P$ $<0.001)$, and $26.78 \%(P<0.001)$, respectively, and the increase in HDL was $7.8 \%(P=$ 0.03 ). It was observed that the decrease in serum TC, LDL-C and triglyceride levels were significantly higher in the second group than the first group. With atorvastatin, the distensibility coefficient (DC) and compliance coefficient (CC) increased from $18.7 \pm 3.4$ to $21.3 \pm 2.910^{-3} \cdot \mathrm{kPa}^{-1}(P<0.001)$ and from $0.69 \pm 0.05$ to $0.77 \pm 0.03 \mathrm{~mm}^{2} \cdot \mathrm{kPa}^{-1}(P<$ $0.001)$ in the first group while they changed from $18.3 \pm 3.6$ to $21.9 \pm 3.010^{-3} \cdot \mathrm{kPa}^{-1}(P$ $<0.001)$ and from $0.70 \pm 0.04$ to $0.81 \pm 0.01 \mathrm{~mm}^{2} \cdot \mathrm{kPa}^{-1}(P<0.001)$ respectively, in the second group. DC and CC increased in both groups, but the differences between the groups were not significant. High doses of atorvastatin reduce blood lipid levels more than conventional doses, however, the change in compliance is not dose-dependent. As endothelial dysfunction is regarded as an early feature of atherosclerosis, there would be no need to administer aggressive doses in a patient without any risk factors other than hyperlipidemia. (Jpn Heart J 2003; 44: 953-961)
\end{abstract}

Key words: Arterial compliance, Atorvastatin

From the Departments of ${ }^{1}$ Internal Medicine, ${ }^{2}$ Family Medicine, ${ }^{3}$ Public Health, ${ }^{4}$ Radiology, and ${ }^{5}$ Cardiology, Cerrahpasa Medical Faculty, Istanbul University, Istanbul, Turkey.

Address for correspondence: Yesari Karter, MD, the Department of Internal Medicine, Cerrahpasa Medical Faculty, Istanbul University, Cerrahpasa Tip Fak.Ic Hastaliklari Anabilim Dali. Cerrahpasa- Istanbul, Turkey.

Received for publication January 6, 2003.

Revised and accepted April 10, 2003. 
$\mathrm{I}_{\mathrm{T}}$ is known that the atherogenic process begins early in life, but there is a long latent period before the appearance of the first clinical signs of arterial disease, such as angina or myocardial infarction. Detection of early changes in atherosclerosis would allow the early treatment and prevention of cardiovascular disease. ${ }^{1)}$ Before any evidence of morphological lesions or plaques, endothelial dysfunction, such as impairment in endothelium dependent vascular tone regulation may occur. ${ }^{2)}$ Vascular distensibility or arterial compliance decreases gradually. ${ }^{3,4)}$ This endothelial dysfunction is regarded as an early feature of atherosclerosis. ${ }^{5)}$ Arterial distensibility can be studied noninvasively in vivo and measures of arterial stiffness have been proposed as surrogate markers for atherosclerosis. ${ }^{6,7)}$

LDL is a major risk factor for atherosclerosis. It was reported that high levels of LDL cause a decrease in arterial compliance and administration of atorvastatin lowers the cholesterol level and reduces the prevalence of atherosclerotic events. $^{6,7)}$

There have been studies on the effects of atorvastatin on serum lipid levels and arterial compliance. Some investigators administered conventional doses while some prefer aggressive therapy, but there is no study comparing these different doses according to their effects on arterial compliance.

In the current study, all other risk factors other than hyperlipidemia were excluded and the effects of conventional doses on lipid levels and arterial compliance were compared with high doses. Our aim was to determine whether the decrease in lipid levels correlated with the increase in arterial compliance and if these changes were dose-dependent.

\section{Methods}

Twenty-four hypercholesterolemia cases (15 men, 9 women) were enrolled in the present double-blind, randomized study. Twelve patients ( 7 males, 5 females; group I) received three months of treatment with $20 \mathrm{mg} /$ day of atorvastatin, while the other 12 patients ( 8 males, 4 females; group II) received $80 \mathrm{mg}$ / day.

Patients with an LDL-C value greater than $170 \mathrm{mg} / \mathrm{dL}$ were included. They had no other risk factors for atherosclerotic vascular disease, and had never received hypolipidemic treatment. The exclusion criteria were hypertension (> 140/90), any signs or symptoms of atherosclerotic vascular disease, diabetes mellitus and other endocrine disorders, obesity (BMI > 27), drugs that may affect lipid metabolism, smoking, and alcoholism. All of the female participants were postmenopausal and none were taking hormone replacement therapy. Patients with elevated liver enzymes and with known sensitivity to drugs were also excluded. 
At baseline, ambulatory blood pressure, arterial compliance of the common carotid artery (CCA), fasting lipid profile, and cardiac structure and function by echocardiography were determined in all patients.

Ambulatory blood pressure was measured using the A and D company limited TM-2421 ambylotory blood pressure recorder which is recommended by the British Society of Hypertension and European Society of Hypertension. ${ }^{8}$

Ultrasonographic examinations were performed in a quiet, temperature-controlled room $\left(22^{\circ} \mathrm{C}\right)$. All patients rested for 20 minutes before the examination. The examinations were all done between $8^{30}$ and $10^{00} \mathrm{AM}$ after overnight fasting. They were performed with a color Doppler ultrasound unit (Siemens Elegra, Erlangen, Germany) equipped with a 4-7.5 MHz lineer transducer, $\mathrm{MHz}$ transducer. Patients were examined in the supine position. All ultrasonographic measurements were performed by an experienced radiologist who was blinded to the grouping of the patients. B-mode ultrasound scans of the left common carotid arteries were performed with this technique. The lumen diameter of the common carotid artery, one centimeter proximal to the bulb, was measured as the distance between intimae-blood interfaces anteriorly and intimae-blood interfaces posteriorly. All measurements were made at the time of scanning on frozen images of longitudinal scans using the electronic calipers of the machine. Systolic and diastolic lumen diameters were taken separately using the cine-mode and the difference between them was estimated (Figure).

The difference between the anterior and posterior wall represented arterial distension, which was the change in diameter during one heart cycle. Data for arterial diastolic diameter $(\mathrm{D})$ and distension $(\Delta \mathrm{D})$ were obtained for each heart beat. Brachial blood pressure was measured with a semiautomatic oscillometric device (Dinamap). Pulse pressure was defined as systolic minus diastolic pressure $(\Delta \mathrm{P})$. Vessel wall properties were calculated according to the following equations:

Distensibility coefficient $(\mathrm{DC})=(2 \Delta \mathrm{D} / \mathrm{D}) / \Delta \mathrm{P}\left(\right.$ in $\left.10^{-3} \cdot \mathrm{kPa}^{-1}\right)$

Compliance coefficient $\left.(\mathrm{CC})=\pi \mathrm{D} \Delta \mathrm{D} /(2 \Delta \mathrm{P})\left(\text { in } \mathrm{mm}^{2} \cdot \mathrm{kPa}^{-1}\right)^{9,10}\right)$

Each measurement was repeated three times on one occasion and the mean value for each measurement was calculated. The measurements of 10 different patients were obtained twice ( 2 hour interval) and the repeatability coefficients (RC) values were $0.35 \mathrm{~mm}$ for systolic diameter and $0.18 \mathrm{~mm}$ for diastolic diameter, which were not statistically different from zero. ${ }^{11)}$

Baseline and posttreatment lipid determinations were done. Blood was collected from the antecubital vein after an overnight fast. Serum total cholesterol 

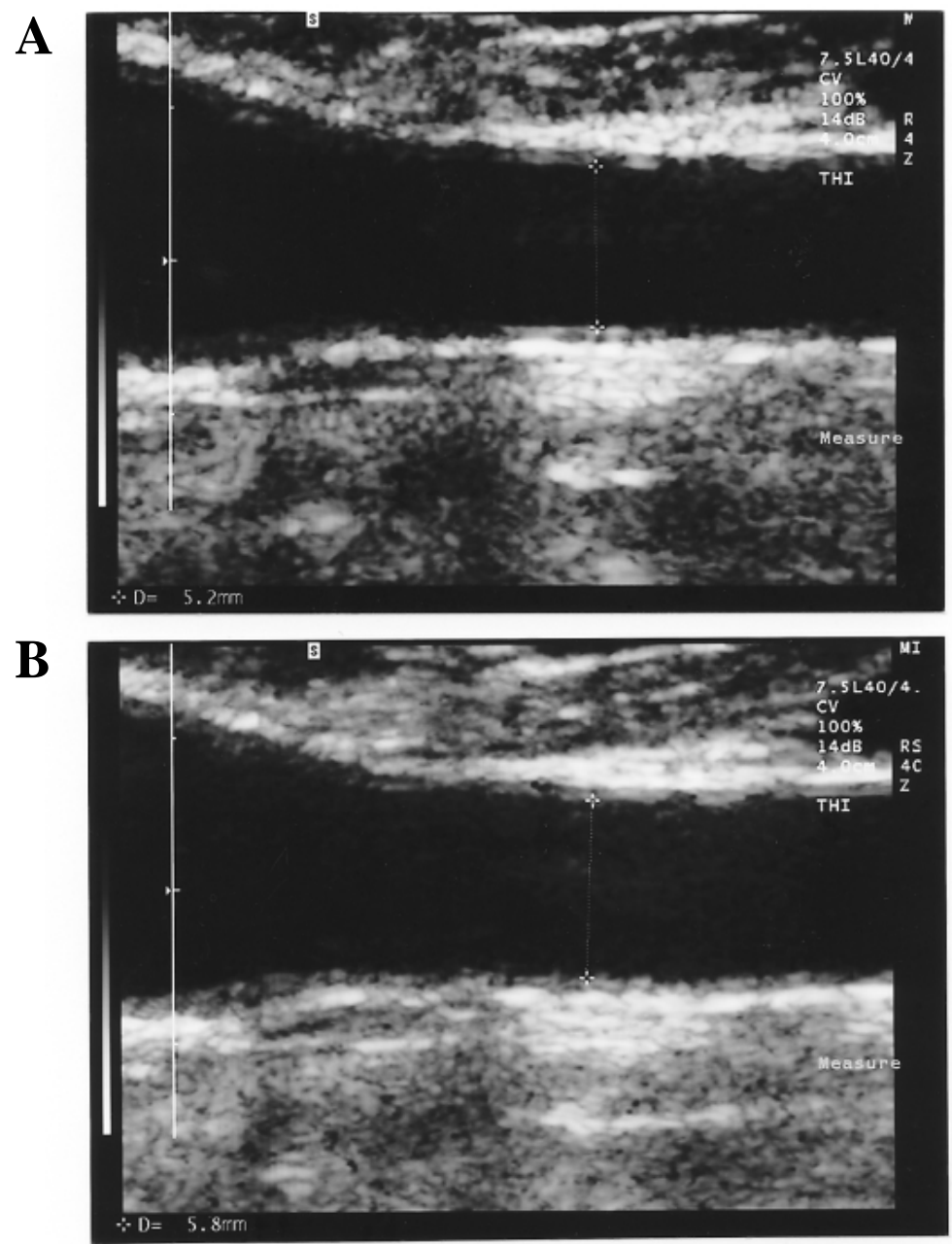

Figure. Left common carotid artery measured $5.2 \mathrm{~mm}$ in diameter during diastole (A) and 5-8 $\mathrm{mm}$ in diameter during systole (B). The change in diameter during one heart cycle was used in the compliance estimation.

(TC), HDL-C, triglycerides (TG), ALT, AST, and CPK values were measured using standard enzymatic methods with a fully automated analyzer (Olympus AU-800). LDL-C was calculated. ${ }^{12)}$

Indexes of cardiac structure and function were assessed at baseline only. Left ventricular end-diastolic posterior wall thickness, interventricular septal wall thickness at end-diastole, left ventricular internal end-diastolic diameter, (LVIDD), and left ventricular internal end-systolic diameter (LVISD) were mea- 
sured using the American Society of Echocardiography convention from Mmode images of the left ventricle, generated in the short axis view at the level of the mitral chordae (2.5 MHz; Hewlett Packard 2500). Using these measurements, left ventricular mass (LVM) and left ventricular mass index (LVMI) were calculated. ${ }^{13,14)}$

Statistical values are expressed as the mean \pm SD. Comparisons within groups were evaluated by the paired $t$ test and between groups by Student's $t$ test. Statistical significance was accepted at the $96 \%$ confidence level.

\section{RESULTS}

The 24 patients comprised a relatively homogenous group in that 12 of them receiving the $20 \mathrm{mg}$ dose and the other 12 receiving $80 \mathrm{mg}$ had similar relevant characteristics such as age, gender distribution, BMI, and blood pressure. More importantly, they had similar plasma TC, TG, LDL-C, and HDL-C levels (Table I).

Blood pressures did not change on average from baseline values and were similar with $20 \mathrm{mg}$ and $80 \mathrm{mg}$. The baseline and last systolic pressure values in group I ( $20 \mathrm{mg}$ ) were $126.9 \pm 6.5$ and $125.7 \pm 5.8 \mathrm{mmHg}$ while they were 128.8 \pm 3.4 and $127.8 \pm 4.1 \mathrm{mmHg}$ in group II $(80 \mathrm{mg})$, respectively. The diastolic values were $81.5 \pm 3.4$ versus $80.0 \pm 5.0 \mathrm{mmHg}$ in group I and $83.5 \pm 2.9$ versus 81.7 \pm 2.8 in group II (Tables II and III).

Treatment with $20 \mathrm{mg}$ of atorvastatin reduced the TC, LDL-C, and TG levels by $32 \%(P<0.001), 40.8 \%(P<0.001)$, and $19 \%(P<0.001)$, respectively. In

Table I. Clinical Characteristics of the Two Study Groups

\begin{tabular}{lcc}
\hline & $\begin{array}{c}\text { Group I } \\
(n=12)\end{array}$ & $\begin{array}{c}\text { Group II } \\
(n=12)\end{array}$ \\
\hline Gender (male/female) & $7 / 5$ & $8 / 4$ \\
Age & 50 & 52 \\
Body mass index $\left(\mathrm{kg} / \mathrm{m}^{2}\right)$ & 25.1 & 24.7 \\
LVMI* $\left(\mathrm{g} / \mathrm{m}^{2}\right)$ & 78.92 & 80.25 \\
Total cholesterol (mg/dL) & $279.7 \pm 18.5$ & $286.9 \pm 13.4$ \\
LDL-C (mg/dL) & $200.0 \pm 18.1$ & $205.3 \pm 16.1$ \\
HDL-C (mg/dL) & $49.3 \pm 6.7$ & $52.3 \pm 4.6$ \\
Triglycerides (mg/dL) & $172.3 \pm 20.7$ & $179.8 \pm 34.3$ \\
Systolic pressure (mmHg) & $126.9 \pm 6.5$ & $128.8 \pm 3.4$ \\
Diastolic pressure (mmHg) & $81.5 \pm 3.4$ & $83.5 \pm 2.9$ \\
& & \\
*Left ventricular mass index & & \\
\hline
\end{tabular}


Table II. Effects of $20 \mathrm{mg}$ of Atorvastatin on Lipid Profile, Blood Pressure, and Arterial Compliance (group I)

\begin{tabular}{lccc}
\hline & Baseline & After treatment & $P$ \\
\hline Total cholesterol $(\mathrm{mg} / \mathrm{dL})$ & $279.7 \pm 18.5$ & $190.2 \pm 10.7$ & $<0.001$ \\
LDL-C $(\mathrm{mg} / \mathrm{dL})$ & $200.0 \pm 18.1$ & $118.3 \pm 11.7$ & $<0.001$ \\
HDL-C $(\mathrm{mg} / \mathrm{dL})$ & $49.3 \pm 6.7$ & $52.75 \pm 6.1$ & 0.002 \\
Triglycerides $(\mathrm{mg} / \mathrm{dL})$ & $172.3 \pm 20.7$ & $139.5 \pm 16.3$ & $<0.001$ \\
Systolic pressure (mmHg) & $126.9 \pm 6.5$ & $125.7 \pm 5.8$ & 0.023 \\
Diastolic pressure (mmHg) & $81.5 \pm 3.4$ & $80.0 \pm 5.0$ & 0.772 \\
$*$ DC $\left(10^{-3} \cdot \mathrm{kPa}^{-1}\right)$ & $18.7 \pm 3.4$ & $21.3 \pm 2.9$ & $<0.001$ \\
$* * \mathrm{CC}\left(\mathrm{mm}^{2} \cdot \mathrm{kPa}^{-1}\right)$ & $0.69 \pm 0.05$ & $0.77 \pm 0.03$ & 0.010 \\
\hline
\end{tabular}

*Distensibility coefficient **Compliance coefficient.

Data are presented as the mean \pm SD.

Table III. Effects of $80 \mathrm{mg}$ of Atorvastatin on Lipid Profile, Blood Pressure, and Arterial Compliance (group II)

\begin{tabular}{lccc}
\hline & Baseline & After treatment & $P$ \\
\hline Total cholesterol (mg/dL) & $286.9 \pm 13.4$ & $176.5 \pm 12.3$ & $<0.001$ \\
LDL-C (mg/dL) & $205.3 \pm 16.1$ & $110.4 \pm 9.1$ & $<0.001$ \\
HDL-C (mg/dL) & $52.3 \pm 4.6$ & $56.3 \pm 5.1$ & 0.03 \\
Triglycerides (mg/dL) & $179.8 \pm 34.3$ & $131.7 \pm 19.3$ & $<0.001$ \\
Systolic pressure (mmHg) & $128.8 \pm 3.4$ & $127.8 \pm 4.1$ & 0.144 \\
Diastolic pressure (mmHg) & $83.5 \pm 2.9$ & $81.7 \pm 2.8$ & 0.368 \\
$*$ DC $\left(10^{-3} \mathrm{k} . P a^{-1}\right)$ & $18.3 \pm 3.6$ & $21.9 \pm 3.0$ & $<0.001$ \\
$* *$ CC $\left(\mathrm{mm}^{2} . \mathrm{kPa}^{-1}\right)$ & $0.70 \pm 0.04$ & $0.81 \pm 0.01$ & $<0.001$ \\
\hline
\end{tabular}

*Distensibility coefficient $* *$ Compliance coefficient.

Data are presented as the mean \pm SD.

the high dose group (group II), these changes were 38.5\% $(P<0.001), 46.2 \%(P$ $<0.001)$, and $26.78 \%(P<0.001)$, respectively. HDL-C increased by $6.9 \%(P=$ $0.002)$ in group I and by $7.8 \%(P=0.03)$ in group II (Tables II and III).

DC and CC were higher in both groups after the treatment. In group I, DC increased from $18.7 \pm 3.4$ to $21.3 \pm 2.910^{-3} \cdot \mathrm{kPa}^{-1}(P<0.001)$ while it increased from $18.3 \pm 3.6$ to $21.9 \pm 3.010^{-3} \cdot \mathrm{kPa}^{-1}(P<0.001)$ in group II. CC also increased from $0.69 \pm 0.05$ to $0.77 \pm 0.03 \mathrm{~mm}^{2} \cdot \mathrm{kPa}^{-1}(P=0.01)$ and from $0.70 \pm 0.04$ to 0.81 $\pm 0.01 \mathrm{~mm}^{2} \cdot \mathrm{kPa}^{-1}(P<0.001)$ in group I and II, respectively (Tables II and III).

The differences in TC $(P<0.001)$, LDL-C $(P=0.002)$, and TG $(P=0.008)$ between the groups were significant, while the differences in HDL-C $(P=0.078)$, DC $(P=0.172)$, and CC $(P=0.761)$ were not (Table IV). 
Table IV. Comparison of the Differences between the Groups After Atorvastatin Treatment

\begin{tabular}{lccr}
\hline & $\begin{array}{c}\text { Differences (Group I) } \\
\text { Mean } \pm \text { SD }\end{array}$ & $\begin{array}{c}\text { Differences (Group II) } \\
\text { Mean } \pm \text { SD }\end{array}$ & $P$ \\
\hline Total cholesterol (mg/dL) & $-89.5 \pm 8.9$ & $-110.1 \pm 7.6$ & $<0.001$ \\
LDL-C (mg/dL) & $-82.5 \pm 8.1$ & $-95.7 \pm 9.9$ & 0.002 \\
HDL-C (mg/dL) & $3.4 \pm 1.1$ & $4.1 \pm 7.4$ & 0.078 \\
Triglycerides (mg/dL) & $-32.8 \pm 6.3$ & $-49 \pm 18.1$ & 0.008 \\
$*$ DC $\left(10^{-3} \cdot \mathrm{kPa}^{-1}\right)$ & $2.58 \pm 4.31$ & $2.75 \pm 0.75$ & 0.896 \\
$* * C C\left(\mathrm{~mm}^{2} \cdot \mathrm{kPa}^{-1}\right)$ & $0.13 \pm 0.15$ & $0.11 \pm 0.04$ & 0.562 \\
\hline
\end{tabular}

*Distensibility coefficient, $* *$ Compliance coefficient.

\section{DiscuSSION}

The relationship between serum lipid levels and arterial compliance was investigated and the effects of conventional and high dose lipid lowering treatments on serum lipid levels and arterial compliance were compared.

Atorvatatin reduced the serum lipid levels and this reduction was dosedependent. In other words, $80 \mathrm{mg}$ of atorvastatin was significantly more effective than $20 \mathrm{mg}$ at reducing serum lipid levels $(38.5 \%, 46.2 \%$. 26.78\% vs $32 \%$, $40.8 \%, 19 \%)$.

DC and CC increased in both groups after the treatment. There was no significant difference between the groups. The change in arterial compliance did not increase by increasing the drug dose. Therefore, the change in arterial compliance was not dose-dependent.

In all published studies, it was concluded that both conventional and high doses caused an increase in arterial compliance in addition to the reduction in serum lipid levels, ${ }^{13,15,16)}$ however, there is no study comparing the effects of different doses on arterial compliance.

The integrity of the endothelium is impaired in patients with atherosclerosis or hypercholesterolemia, presumably because of a direct injurious effect of elevated levels of LDL-C on the endothelium. ${ }^{17)}$ Moreover, hypercholesterolemia has been suggested to stimulate the generation of superoxide radicals by the endothelium. Superoxide directly inactivates NO and may also increase the subsequent oxidation of LDL particles by the formation of peroxynitrite. A reduction in serum cholesterol is associated with the normalization of oxygen-derived free radical production. ${ }^{18)}$ Thus, a decrease in free radical production and consecutively less degradation together with increased synthesis of NO can explain the observed improvement in the bioavailability of NO and thus in endotheliumdependent vasodilatation in the patients. ${ }^{19)}$ It is clear that NO is the effective fac- 
tor in increasing arterial compliance, but it is not clear whether conventional doses are sufficient or if aggressive lipid lowering doses are necessary to achieve a better or a target arterial compliance. O'Driscol, et al reported that the improvement in endothelial function with simvastatin therapy did not correlate with the decrease in serum cholesterol. ${ }^{20)}$

Before any evidence of morphological lesions or plaques, endothelial dysfunctions such as impairment in endothelium-dependent vascular tone regulation may occur. Vascular distensibility or arterial compliance decrease gradually. This endothelial dysfunction is regarded as an early feature of atherosclerosis. Arterial distensibility can be studied noninvasively in vivo and measures of arterial stiffness have been proposed as surrogate markers for atherosclerosis. Therefore, a decrease in arterial compliance can be taken as the starting point of atherosclerosis. The main purpose should be to prevent the progression of arterial stiffness.

Of course lipid levels, which are the main factors in the progression of atherosclerosis, must be lowered, but is it necessary to use aggressive doses in patients without any known risk factors except severe hyperlipidemia.

In our study, we saw that arterial compliance increased with both $20 \mathrm{mg}$ and $80 \mathrm{mg}$ of atorvastatin; however, the change in arterial compliance between these two groups was not significantly different. As it is known that arterial stiffness increases due to impairment of NO by elevated LDL, we can conclude that low doses of atorvastatin are sufficient to overcome the effect of free radicals on NO bioavailability.

The adverse effects of a drug are also important and increase with an increase in dose. The incidence of hepatotoxicity with conventional doses was estimated to be $0.7 \%$ while it was $2.3 \%$ with aggressive doses. ${ }^{21)}$ It is also clear that the cost increases as the dose increases. As there was no significant difference in the change in compliance, it does not seem right to spend more and increase the risk of adverse effects due to administration of high doses in patients without any risk factors other than hyperlipidemia.

\section{REFERENCES}

1. Toikka JO Niemi P, Ahotupo M, et al. Large-artery elastic properties in young man: Relationships to serum lipoproteins and oxidized low-density lipoproteins. Arterioscler Thromb 1999; 19: 436-41.

2. Arcaro G, Zenere BM, Travia D, et al. Non-invasive detection of early endothelial disfunction in hypercholesterolaemic subjects. Atherosclerosis 1995; 114: 247-54.

3. Lehmann ED, Hopkins KD, Gosling RG. Aortic compliance measurements using Doppler ultrasound. In vivo biochemical correlates. Ultrasound Med Biol 1993; 19: 683-710.

4. Salomaa V, Riley W, Kark JD, Nardo C, Folsom AR. Non insulin dependent diabetes mellitus and fasting glucose and insulin concentrations are associated with arterial stiffness indexes: the ARIC Study: Atherosclerosis Risk in Communities Study. Circulation 1995; 91: 1432-43. 
5. de Man FH, Weverling-Rijnsburger AW, van der Laarse A, Smelt AH, Jukema JW, Blauw GJ. Not acute but chronic hypertriglyceridemia is associated with impaired endothelium-dependent vasodilatation. Arterioscler Thromb 2000; 20: 744-56.

6. Dart AM, Lacombe F, Yeoh JK, et al. Aortic distensibility in patients with isolated hypercholesterolaemia, coronary artery disease or cardiac transplantation. Lancet 1991; 338: 270-3.

7. Arnett DK, Ewans GW, Riley WA. Arterial stiffness: a new cardiovascular risk factor? Am J Epidemiol 1994; 140: 669-82.

8. O'Brien E, Coats A, Owens $\mathrm{P}$, et al. Use and interpretation of ambulatory blood pressure monitoring: recommendations of the British Hypertension Society. BMJ 2000; 320: 1128-34.

9. Smilde TJ, Van den Berkmortel FW, Boers GHJ, et al. Carotid and femoral artery wall thickness and stiffness in patients at risk for cardiovascular disease, with special emphasis on hyperhomocysteinemia. Arterioscler Thromb 1998; 18: 1958-63.

10. Hoeks AP, Brands PJ, Smeets FA, Reneman RS. Assessment of the distensibility of superficial arteries. Ultrasound Med Biol 1990; 16: 121-8.

11. Aggoun Y, Bonnet D, Sidi D, et al. Arterial mechanical changes in children with familial hypercholesterolemia. Arterioscler Thromb Vasc Biol 2000; 20: 2070-5.

12. Friedewald WT, Levy RI, Fredrickson DS. Estimation of the concentration of low density lipoprotein cholesterol in plasma without the use of the ultracentrifuge. Clin Chem 1972; 18: 499-502.

13. Ferrier KE, Muhlmann MH, Baguet JP, et al. Intensive cholesterol reduction lowers blood pressure and large artery stiffness in isolated systolic hypertension. J Am Coll Cardiol. 2002; 39: 1020-5.

14. Devereux RB, Alonso DR, Lutas EM, et al. Echocardiographic assessment of left ventricular hypertrophy: comparison to necropsy findings. Am J Cardiol 1986; 57: 450-8.

15. Shige H, Dart A, Nestel P. Simvastatin improves arterial compliance in the lower limb but not in the aorta. Atherosclerosis. 2001; 155: 245-50.

16. Leibovitz E, Hazanov N, Zimlichman R, Shargorodsky M, Gavish D. Treatment with atorvastatin improves small artery compliance in patients with severe hypercholesterolemia. Am J Hypertens 2001; 14: 1096-8.

17. Seiler C, Hess OM, Buechi M, Suter TM, Krayenbuehl HP. Influence of serum cholesterol and other coronary risk factors on vasomotion of angiographically normal coronary arteries. Circulation 1993; 88: 2139-48.

18. Ohara Y, Peterson TE, Harrison DG. Dietary treatment of hypercholesterolemia normalizes endothelial superoxide anion production. Circulation 1993; 88: 467. Abstract.

19. John S, Schlaich M, Langenfeld M, et al. Increased bioavailability of nitric oxide after lipid lowering therapy in hypercholesterolemic patients. Circulation 1998; 98: 211-6.

20. O'Driscol G, Green D, Taylor RR. Simvastatin, an HGM-coenzyme A reductase inhibitor, improves endothelial function within 1 month. Circulation 1997; 95: 1126-31.

21. Black DM, Bakker RG, Nawrocki JW. An overview of the clinical safety profile of atorvastatin (lipitor), a new HMG-CoA reductase inhibitor. Arch Intern Med 1998; 158: 577-84. 